\title{
Altered microRNAs related to synaptic function as potential plasma biomarkers for Alzheimer's disease
}

Dolores Siedlecki-Wullich 1,2, Judit Català-Solsona ${ }^{1,2}$, Cristina Fábregas ${ }^{1,2}$, Isabel Hernández ${ }^{3}$, Jordi Clarimon 2,4, Alberto Lleó ${ }^{2,4}$, Merce Boada ${ }^{2,3}$, Carlos A. Saura ${ }^{1,2}$, José Rodríguez-Álvarez ${ }^{1,2,5^{*}}$ (D) and Alfredo J. Miñano-Molina ${ }^{1,2^{*}}$

\begin{abstract}
Background: Several evidences suggest that failure of synaptic function occurs at preclinical stages of Alzheimer's disease (AD) preceding neuronal loss and the classical AD pathological hallmarks. Nowadays, there is an urgent need to identify reliable biomarkers that could be obtained with non-invasive methods to improve AD diagnosis at early stages. Here, we have examined plasma levels of a group of miRNAs related to synaptic proteins in a cohort composed of cognitive healthy controls $(\mathrm{HC})$, mild cognitive impairment (MCl) and $\mathrm{AD}$ subjects.
\end{abstract}

Methods: Plasma and brain levels of miRNAs were analysed in two different cohorts including $38 \mathrm{HC}, 26 \mathrm{MCl}$, $56 \mathrm{AD}$ dementia patients and 27 frontotemporal dementia (FTD) patients. D'Agostino and Pearson and Shapiro-Wilk tests were used to evaluate data normality. miRNA levels between groups were compared using a two-sided nonparametric Mann-Whitney test and sensitivity and specificity was determined by receiver operating characteristic curve analysis.

Results: Significant upregulation of miR-92a-3p, miR-181c-5p and miR-210-3p was found in the plasma of both $\mathrm{MCl}$ and $\mathrm{AD}$ subjects. $\mathrm{MCl}$ patients that progress to $\mathrm{AD}$ showed higher plasma levels of these miRNAs. By contrast, no changes in miR-92a-3p, miR-181c-5p or miR-210-3p levels were observed in plasma obtained from a cohort of FTD.

Conclusion: Our study shows that plasma miR-92a-3p, miR-181c-5p and miR-210-3p constitute a specific molecular signature potentially useful as a potential biomarker for AD.

Keywords: Alzheimer's disease, Mild cognitive impairment, Synapses, miRNAs, Plasma, Human, Biomarker, Frontotemporal dementia

\section{Background}

It is estimated that more than 40 million people worldwide are affected by Alzheimer's disease (AD) and it is expected that about 100 million could be affected by 2050 . Unfortunately, there is no treatment to prevent or even reverse $\mathrm{AD}$, making the finding of new therapeutic breakthroughs a huge challenge for our societies [1]. During the last decade, much effort has been devoted to the establishment of early $\mathrm{AD}$ biomarkers that could help to

\footnotetext{
* Correspondence: jose.rodriguez@uab.cat; alfredo.minano@uab.cat ${ }^{1}$ Institut de Neurociències and Dpt. Bioquímica i Biología Molecular, Universitat Autònoma de Barcelona, 08193 Cerdanyola del Vallès, Spain Full list of author information is available at the end of the article
}

implement appropriate personalised care programmes before the onset of neurodegeneration and dementia. Moreover, effective early AD biomarkers could improve the design of clinical trials and help to reverse the continuous failures in the search of disease-modifying agents for $\mathrm{AD}$ obtained in the last years [2-4].

The best AD biomarkers achieved to date are based on neuroimaging methods $(A \beta$ in the brain or hippocampal atrophy) or by the detection in the cerebrospinal fluid (CSF) of total tau, phospho-tau and $\mathrm{A} \beta_{42}$ [5-8]. Recently, a specific roadmap has been suggested to validate these neuroimaging- and CSF-derived biomarkers [9]. Unfortunately, all these potential biomarkers could not

(c) The Author(s). 2019 Open Access This article is distributed under the terms of the Creative Commons Attribution 4.0 International License (http://creativecommons.org/licenses/by/4.0/), which permits unrestricted use, distribution, and 
be used in routine clinical screening due to their invasiveness and economic limitations.

On the other hand, several evidences indicate that cognitive impairment observed in early stages of $A D$ could be explained by alterations in synaptic function that precedes neurodegeneration [10]. Furthermore, some reports have shown that deregulation of synaptic proteins could be related to early cognitive dysfunction in experimental models of AD [11-14]. Thus, changes in the regulatory mechanisms involved in the expression of synaptic proteins could be valuable for assessing prognosis and the rate of cognitive decline in $\mathrm{AD}$.

MicroRNAs (miRNAs) are small non-coding RNAs that play a significant role in local control of mRNA translation. In fact, a single miRNA could regulate the local expression of multiple proteins. Several studies have shown that some miRNAs control the formation, maturation and function of synapses [15] and alteration in their levels could underlie synaptic dysfunction in pathological states [16]. Notably, a number of specific miRNAs are misregulated in AD, including miRNAs implicated in the regulation of key genes involved in AD, such as APP or BACE1, or neuronal function such as glutamate receptors [17-20]. Extensive interest has been focused on identifying changes in specific miRNAs that could be used as AD biomarkers [21-25]. miRNAs can be found in the blood where they are transported in different structures that protect them from degradation $[26,27]$. Since blood collection is an easy and non-invasive procedure, the identification of blood-based miRNA biomarkers for $\mathrm{AD}$ has drawn attention during the last years [28]. Altered levels of certain miRNAs in plasma of $\mathrm{AD}$ patients have been previously reported [21, 25, 29-32]; however, few data exist about changes in plasma levels of miRNAs that are related to synaptic protein function in $\mathrm{AD}$ [33]. In the present study, we have analysed several miRNAs related to synaptic proteins in plasma samples from mild cognitive impairment (MCI) and $\mathrm{AD}$ subjects. We have also monitored plasma samples from frontotemporal dementia (FTD) patients to verify the specificity of the obtained results. Our data suggest that miR-92a-3p, miR-181c-5p and miR-2 10-3p are potential and specific plasma biomarkers for $\mathrm{AD}$.

\section{Methods}

\section{Subjects}

Plasma samples analysed in this study were obtained from two different cohorts. The first cohort provided by Fundació ACE (Barcelona, Spain) includes 14 HC, $26 \mathrm{MCI}$ and $56 \mathrm{AD}$ dementia patients. The second cohort was recruited at the Memory Unit of the Hospital Sant Pau (Barcelona, Spain) and consists of $24 \mathrm{HC}$ and 27 FTD patients. Participants were clinically diagnosed by neurologists and classified according to internationally accepted diagnostic criteria [34]. Specifically, MCI subjects fulfilled Petersen's diagnostic criteria [35], and their neuropsychological assessment was done by the previously validated NBACE battery tests [36]. FTD participants include 19 patients with possible or probable behavioural variant [37] and 6 with semantic variant of primary progressive aphasia [38]. Two patients with FTD were additionally diagnosed with concomitant ALS according to El Escorial criteria [39]. Demographic and clinical characteristics of cohorts 1 and 2 are summarised in Table 1. Brain tissue samples were provided by 3 different Spanish centres: Fundación Cien, Hospital Clinic-IDIBAPS and Hospital Universitario Fundación Alcorcón. Demographic and clinical characteristics of the subjects are listed in Additional file 1: Table S1. Brain tissue from entorhinal cortex $(n=13)$, hippocampus $(n=49)$ and cerebellum $(n=$ 30) was analysed.

\section{Sample processing, RNA extraction and reverse transcription}

Blood samples were collected in EDTA-containing tubes, as recommended [40]. After $20 \mathrm{~min}$ centrifugation $(2500 \times g)$, plasma was separated, aliquoted and stored at $-80^{\circ} \mathrm{C}$ until use. Plasma samples were thawed on ice for RNA extraction, and hemolysis of each sample was analysed at the time measuring absorbance at $414 / 375 \mathrm{~nm}$ (414/375 ratio > 1.4 were considered hemolysed) [41]. RNA was isolated from $200 \mu \mathrm{L}$ of plasma, using the miRNeasy RNA isolation kit (Qiagen) following the manufacturer's indications. Two microlitres of RNA was reverse-transcribed to cDNA using TaqMan ${ }^{\text {tow }}$ Advanced miRNA cDNA Synthesis Kit (Thermo Fisher Scientific).

Table 1 The expression levels of miRNAs related to synaptic proteins

\begin{tabular}{|c|c|c|c|c|c|}
\hline \multicolumn{4}{|l|}{ Cohort 1} & \multicolumn{2}{|l|}{ Cohort 2} \\
\hline & $\mathrm{HC}$ & $\mathrm{MCl}$ & $A D$ & $\mathrm{HC}$ & FTD \\
\hline Cohort size & 14 & 26 & 56 & 24 & 27 \\
\hline Men/women & $7 / 7$ & $10 / 16$ & $15 / 41$ & $11 / 13$ & $17 / 10$ \\
\hline Age (years) & $68.29 \pm 8.99$ & $72.0 \pm 8.49$ & $77.77 \pm 6.69$ & $67.03 \pm 5.05$ & $68.87 \pm 7.48$ \\
\hline GDS & $2.07 \pm 0.26$ & $3.15 \pm 0.37$ & $4.64 \pm 1.02$ & $1 \pm 0$ & $3.76 \pm 0.99$ \\
\hline MMSE & $29.21 \pm 1.05$ & $26.92 \pm 2.22$ & $16.05 \pm 7.23$ & $28.5 \pm 1.69$ & $25.5 \pm 3.76$ \\
\hline
\end{tabular}

All data are shown as mean \pm SD

$H C$ cognitively healthy controls, $M C I$ mild cognitive impairment, AD Alzheimer's disease, FTD frontotemporal dementia, MMSE Mini-Mental State Examination, GDS Global Deterioration Scale 
Postmortem brains were quickly homogenated in dry ice to prevent thawing, and RNA was isolated from 20 to $50 \mathrm{mg}$ of tissue from each brain area using miRVana miRNA Isolation Kit (Thermo Fisher Scientific) following the manufacturer's instructions. RNA quality was evaluated using the Agilent 2100 bioanalyzer. Samples with RNA integrity number (RIN) under 4 were excluded [40, 42]. Ten nanogrammes of RNA were reverse-transcribed to cDNA using TaqMan ${ }^{\text {Tu }}$ MicroRNA Reverse Transcription Kit (Thermo Fisher Scientific).

\section{miRNAs quantification by RT-qPCR}

Quantitative real-time PCR (RT-qPCR) was performed from $5 \mu \mathrm{L}$ of $1 / 10$ diluted cDNA (plasma samples) using TaqMan Fast Advanced Master Mix and TaqMan Advanced miRNA Assays (Thermo Fisher Scientific) or from $1.33 \mu \mathrm{L}$ of cDNA (tissue samples) using TaqMan ${ }^{\mathrm{mm}}$ Universal Master Mix II, with UNG. Applied Biosystems 7500 Fast instrument was used for amplification. Samples were run in duplicate, and internal control samples were repeated in every plate to avoid batch effects. Raw Ct data acquired using the 7500 Software v2.0.6 (Applied Biosystems) was exported to LinRegPCR software to calculate the amplification efficiency for each reaction. Reactions with amplification efficiency below 1.6 were discarded. Ct values and average efficiencies obtained from LinRegPCR were used to analyse miRNA levels by the comparative $\Delta \Delta \mathrm{Ct}$ method [43]. To date, there is no consensus on the use of particular reference genes for miRNA level normalisation in AD studies. Therefore, in this study, the stability of some described reference genes was evaluated using the NormFinder algorithm [44]. hsa-miR-191-5p and hsa-miR-484 were identified as the most stable reference genes along with all plasma samples. In addition, hsa-miR-191-5p and hsa-miR-484 showed higher correlation than other candidates (Spearman's correlation coefficient $r=0.89 ; P<0.0001)$. U18 and RNU48 were selected for tissue data normalisation. miRNA levels were normalised versus the geometric mean of selected reference genes, to compensate abundance differences between miRNAs and prevent statistical outliers [45].

\section{Western blotting}

Human brain tissue was lysed in ice with cold RIPA buffer $(50 \mathrm{mM}$ Tris base $\mathrm{pH} 7.4,150 \mathrm{mM} \mathrm{NaCl}, 2 \mathrm{mM}$ EDTA, 1\% NP40, 0.5\% Triton X-100, 0.1\% SDS, $1 \mathrm{mM}$ $\mathrm{Na}_{3} \mathrm{VO}_{4}, 25 \mathrm{mM} \mathrm{NaF}, 1 \mathrm{mM}$ PMSF, $1 / 100$ protease inhibitors and $1 / 100$ phosphatases inhibitors cocktail), sonicated and centrifuged. The supernatant was recovered, and concentration was determined by BCA assay. An equal amount of protein was loaded in $10-12 \%$ polyacrylamide gels and separated by electrophoresis under denaturing conditions (SDS-PAGE). Proteins were then transferred to nitrocellulose membrane (GE Healthcare), and membranes were further incubated with blocking solution (10\% dry milk, 0.1\% BSA, pH 7.4) for $1 \mathrm{~h}$ and washed with phosphate buffer saline-Tween (PBT-T). Next, membranes were incubated with primary antibodies (anti-NPTX1 (1:1000, BD), anti-NPTXR (1:500, Santa Cruz) and anti-b-actin (1:5000, Sigma) overnight at $4{ }^{\circ} \mathrm{C}$. After washing membranes with PBT-T, they were incubated at room temperature for $1 \mathrm{~h}$ with secondary peroxidase-coupled antibodies (mouse or rabbit as needed) prepared in blocking solution. After repeated washes, proteins were detected by chemoluminescence reaction using ECL Western Blotting reagent (GE Healthcare). Immunoblots were analysed by densitometry using ImageJ (National Institutes of Health, Bethesda, MD), and protein levels were corrected for corresponding loading control.

\section{Statistical analysis}

$\mathrm{Ct}$ values were normalised versus the $\mathrm{Ct}$ mean of the control group and $\log 2$ transformed. D'Agostino and Pearson and Shapiro-Wilk tests were used to evaluate data normality. miRNA levels from controls, and patients were compared using a two-sided nonparametric Mann-Whitney test. Bonferroni correction was applied for multiple comparisons when needed. Non-parametric Spearmen's rank correlation test was used to determine the correlation between miRNA expression levels and age. $P$ values $<0.05$ were considered statistically significant. ROC curve analysis under a nonparametric approach was used to obtain the area under de curve (AUC) to evaluate sensitivity and specificity of each miRNA as a predictive biomarker. Logistic regression was applied to evaluate biomarker combination by ROC curve analysis. Statistical analysis was performed using GraphPad Prism software v6.01 (GraphPad Software Inc., CA, USA) and MedCalc (v17.9.7).

\section{Results \\ Upregulation of miR-92a, miR-181c and miR-210 in plasma of $\mathrm{MCl}$ and $\mathrm{AD}$ patients}

The expression levels of miRNAs related to synaptic proteins, miR-92a-3p, miR-181c-5p, miR-210-3p and miR-584-5p (Additional file 1: Table S2), were analysed by RT-qPCR in the plasma of a cohort consisting of 14 $\mathrm{HC}, 26 \mathrm{MCI}$ and 56 sporadic AD subjects (Table 1). We found a significant increase in the levels of miR-92a-3p, miR-181c-5p and miR-210-3p in plasma from AD patients when compared with HC (Fig. 1a; miR-92a-3p: $P$ $=0.0442, \log 2$ fold change $=0.52 ;$ miR-181c $-5 \mathrm{p}: P=$ $0.0024, \log 2$ fold change $=0.67$; miR-210-3p: $P=0.0006$, $\log 2$ fold change $=0.60$ ). A significant increase was also observed in MCI plasma samples for miR-181c-5p $(P=$ $0.0004, \log 2$ fold change $=0.80)$ and miR-210-3p $(P=$ 

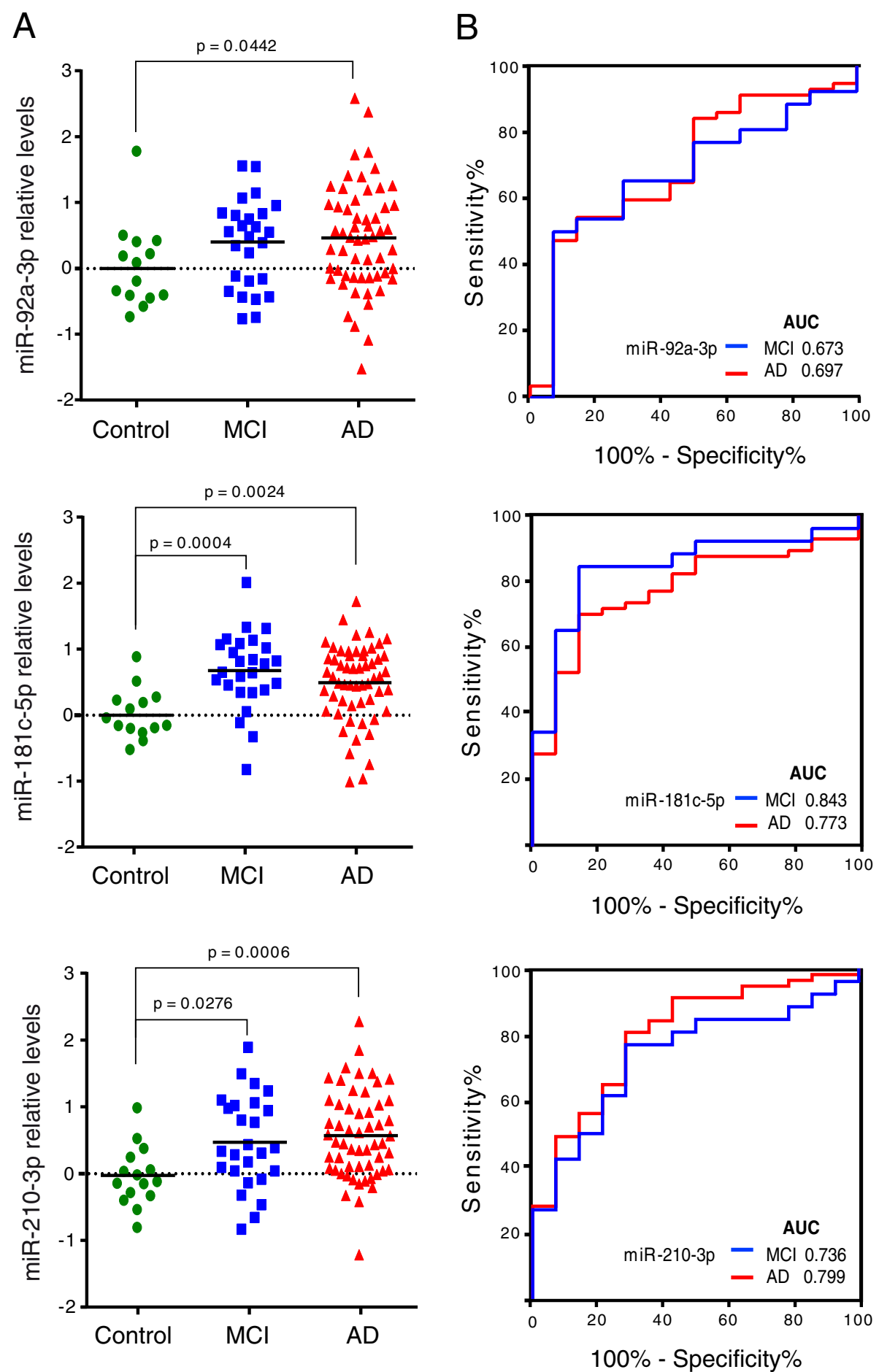

Fig. 1 Circulating miRNA levels at different stages of AD pathology compared with cognitively normal controls (a). Log2 transformed data were normalised versus the geometric mean of miR-191-5p and miR-484 levels. Statistical significance was evaluated by the Mann-Whitney $U$ test followed by the Bonferroni correction for multiple comparisons. $P$ values $<0.05$ were considered statistically significant. $\mathbf{b}$ Receiver operating characteristic (ROC) curve analysis was performed to distinguish $\mathrm{MCl}$ (blue) and $\mathrm{AD}$ (red) cases from healthy controls. The area under the curve (AUC) is shown for each miRNA and stage 
0.0276, $\log 2$ fold change $=0.49)$ whereas an increasing trend was observed for miR-92a-3p $(P=0.0798, \log 2$ fold change $=0.55)$. By contrast, no apparent changes were observed in miR-584-5p expression levels between HC and MCI or AD subjects (data not shown). Statistical correlation analysis revealed that those changes were not due to the increasing age in the $\mathrm{MCI}$ and $\mathrm{AD}$ groups (Additional file 1: Figure S2). Also, no differences between sexes were observed in $\mathrm{HC}, \mathrm{MCI}$ and $\mathrm{AD}$ groups (Additional file 1: Figure S3).

Next, ROC curve analysis was performed to evaluate the diagnostic potential of miR-92a-3p, miR-181c-5p and miR-210-3p (Fig. 1b). The values obtained for miR-92a-3p, miR-181c-5p and miR-210-3p when HC were compared with $\mathrm{MCI}$ subjects were as follows: AUC values of $0.67,0.84$ and 0.74 , respectively; $50.00 \%$, $84.62 \%$ and $76.92 \%$ of sensitivity, respectively; and $92.86 \%, 85.71 \%$ and $71.43 \%$ of specificity, respectively (Table 2). AUC values for miR-92a-3p, miR-181c-5p and miR-210-3p when AD subjects were compared to $\mathrm{HC}$ were $0.70,0.77$ and 0.80 , respectively. Whereas miR-92a-3p has $47.37 \%$ of sensitivity and $92.86 \%$ of specificity, miR-181c-5p has $70.18 \%$ of sensitivity and $85.71 \%$ of specificity and miR-210-3p has $80.70 \%$ of sensitivity and $71.43 \%$ of specificity (Table 3 ). ROC curve analysis using logistic regression for miR-92a-3p, miR-181c-5p and miR-210-3p combination yielded a better diagnostic value for $\mathrm{MCI}$ and $\mathrm{AD}$, suggesting that these miRNAs together could be used as a molecular signature for early diagnosis of $\mathrm{MCI}$ and/or $\mathrm{AD}$. We determined an AUC value of 0.893 , a sensitivity of $84.62 \%$ and a specificity of $85.71 \%$ for distinguishing MCI from HC (Fig. 2a and Table 2). This molecular miRNA signature provided an AUC value of 0.855 , a sensitivity of $92.86 \%$ and a specificity of $71.43 \%$ when AD samples were compared to $\mathrm{HC}$ (Fig. 2b and Table 3).

We also looked for eventual sex-dependent differences in the diagnostic potential of the examined miRNAs
(Fig. 2). We found a slightly better diagnostic value for men than for women to distinguish MCI from HC subjects (Table 2): (a) the AUC values for women and men are 0.82 and 0.96 respectively; (b) $87.50 \%$ sensitivity for women and 100\% for men; and (c) $71.43 \%$ and $85.71 \%$ specificity for women and men, respectively. On the other hand, the values obtained when comparing $\mathrm{AD}$ with $\mathrm{HC}$ subjects indicated a better diagnostic specificity and a lower sensitivity for men than for women (Table 3): (a) the AUC values for women and men are 0.82 and 0.94; (b) $90.24 \%$ and $75.00 \%$ sensitivity for women and men; and (c) $71.43 \%$ and $100 \%$ specificity for women and men, respectively.

In order to provide further support to the value of our molecular miRNA signature as a molecular biomarker for early AD diagnosis, we have followed up 19 of the 26 MCI subjects for 1 to 12 years (Additional file 1: Table S3). Three subjects diagnosed as MCI (11.5\%; P4, P8 and P22) showed no cognitive impairment after 1 (P4 and P22) or 4 (P8) years, and another two (P14 and P18) were still diagnosed as MCI after 3 and 11 years. The rest of the cases, all developed dementia being $\mathrm{AD}$ the most prominent. Only one patient progressed to FTD (P7) and another to vascular dementia (P11; Additional file 1: Table S3). Interestingly, we have observed that all the MCI patients that progressed to $\mathrm{AD}$ have higher values of the miRNA signature compared to the MCI patients that did not evolve to AD (Fig. 3).

\section{Expression levels of miR-92a, miR-181c and miR-210 in AD brains}

To investigate whether the observed changes in miR-92a-3p, miR-181c-5p and miR-210-3p levels were associated to changes in the brain, we measured their levels in the entorhinal cortex, hippocampus and cerebellum from cognitively normal controls (Braak I/II) and early (Braak III/IV) and late (Braak V/VI) AD patients (Additional file 1: Table S1 and Additional file 1: Figure S1). We found that

Table 2 Individual and signature miRNAs performance characteristics in predicting MCI stage. Signature miRNA performance characteristics in women and men

\begin{tabular}{|c|c|c|c|c|c|}
\hline miRNA/signature & $A \cup C$ & Sensitivity \% & Specificity \% & Youden Index J & $P$ value \\
\hline miR-92a-3p & 0.673 & 50.00 & 92.86 & 0.428 & 0.0563 \\
\hline miR-181c-5p & 0.843 & 84.62 & 85.71 & 0.703 & $<0.0001$ \\
\hline miR-210-3p & 0.736 & 76.92 & 71.43 & 0.483 & 0.0038 \\
\hline miR-92a-3p/miR-210-3p & 0.731 & 73.08 & 71.43 & 0.445 & 0.0050 \\
\hline miR-92a-3p/miR-181c-5p & 0.838 & 84.62 & 78.57 & 0.632 & $<0.0001$ \\
\hline miR-181c-5p/miR-210-3p & 0.865 & 88.46 & 78.57 & 0.670 & $<0.0001$ \\
\hline miR-92a-3p/miR-181c-5p/ miR-210-3p & 0.893 & 84.62 & 85.71 & 0.703 & $<0.0001$ \\
\hline Women & 0.821 & 87.50 & 71.43 & 0.589 & 0.0005 \\
\hline Men & 0.957 & 100.0 & 85.71 & 0.857 & $<0.0001$ \\
\hline
\end{tabular}

AUC area under the curve 
Table 3 Individual and signature miRNAs performance characteristics in predicting AD. Signature miRNAs performance characteristics in women and men

\begin{tabular}{|c|c|c|c|c|c|}
\hline miRNA/signature & AUC & Sensitivity \% & Specificity \% & Youden Index J & $P$ value \\
\hline miR-92a-3p & 0.697 & 47.37 & 92.86 & 0.402 & 0.0124 \\
\hline miR-181c-5p & 0.773 & 70.18 & 85.71 & 0.559 & $<0.0001$ \\
\hline miR-210-3p & 0.799 & 80.70 & 71.43 & 0.521 & $<0.0001$ \\
\hline miR-92a-3p/miR-210-3p & 0.807 & 87.72 & 64.29 & 0.520 & $<0.0001$ \\
\hline miR-92a-3p/miR-181c-5p & 0.787 & 84.21 & 71.43 & 0.556 & 0.0001 \\
\hline miR-181c-5p/miR-210-3p & 0.853 & 84.21 & 78.57 & 0.628 & $<0.0001$ \\
\hline miR-92a-3p/miR-181c-5p/ miR-210-3p & 0.855 & 92.86 & 71.43 & 0.644 & $<0.0001$ \\
\hline Women & 0.815 & 90.24 & 71.43 & 0.617 & 0.0009 \\
\hline Men & 0.938 & 75.0 & 100.0 & 0.750 & $<0.0001$ \\
\hline
\end{tabular}

$A U C$ area under the curve

miR-92a-3p, miR-181c-5p and miR-210-3p levels are slightly increased in the entorhinal cortex at early and late $\mathrm{AD}$ compared to $\mathrm{HC}$. However, it only reached statistical significance in the case of miR-92a-3p when compared to late $\mathrm{AD}$ (Braak V-VI; Additional file 1: Figure S1A), probably due to the small number of samples analysed in this region. A similar trend was observed for miR-92a-3p and miR-181c-5p in the hippocampus (Additional file 1: Figure S1B). No significant changes of miR-210-3p in the hippocampus or in miR92a-3p, miR-181c-5p and miR-210-3p in the cerebellum were observed at any disease stage (Additional file 1: Figure S1B, C). Thus, although there is an overall trend to increase in the hippocampus and entorhinal cortex, it is not possible to conclude that miRNA changes observed in plasma are related to those observed in the brain since they lack global statistical significance. miR-181c-5p has neuronal pentraxin 1 (NPTX1) and neuronal pentraxin receptor (NPTXR) as potential targets. Since miR-181c-5p is increased in the entorhinal cortex, we next examined the protein levels of NPTX1 and NPTXR wanted to check whether this increase coincided with a change in the levels of these proteins. As shown in Additional file 1: Figure S3, we observed a decrease in NPTX1 and NPTXR although it did not reach statistical significance likely due to the low number of samples.

\section{Expression levels of miR92a, miR181c and miR210 are not affected in FTD patients}

In order to determine whether the changes observed in miR-92a-3p, miR-181c-5p and miR-210-3p in plasma were specific of MCI and AD subjects, we decided to analyse plasma samples from a cohort of FTD patients. None of the abovementioned miRNAs were altered in FTD (Fig. 4) suggesting that the changes observed could be specific for MCI and AD subjects.

\section{Discussion}

The search of blood-based biomarkers for early detection of $\mathrm{AD}$ has gained increasing attention during the last decade due to the non-invasiveness of the procedure and its low economical costs when compared to the use of CSF or neuroimaging techniques, which would potentially allow their use in routine tests worldwide. Among the diversity of molecules and factors that could be analysed in blood-derived samples, miRNAs represent one of the most promising approaches to identify peripheral $\mathrm{AD}$ biomarkers [7, 17, 20, 46] since they are relatively abundant and highly stable in blood.

Several reports have performed miRNAs profiling in brain and CSF that have identified numerous miRNAs as potential biomarkers to detect AD [46-49]. Whereas some studies have focused on miRNAs regulating specific AD-related proteins, little attention has been devoted to miRNAs involved in the regulation of synaptic proteins. In the present study, we have analysed plasma levels of specific miRNAs related to the regulation of synaptic proteins, especially glutamatergic synapses, in $\mathrm{HC}, \mathrm{MCI}$ and AD patients. Thus, this work complements other reports that have suggested the potential of miRNAs as biomarkers for AD.

From the different miRNAs analysed, we have observed a significant increase in miR-92a-3p, miR-181c-5p and miR-210-3p plasma levels in MCI and AD. Moreover, an increase in the levels of these three miRNAs was observed in AD entorhinal cortex. However, only miR-92a-3p changes reached statistical significance probably due to the low number of available samples used. No significant changes in hippocampal levels were observed for miR-181c-5p and miR-210-3p. Hence, our data adds on many previous reports indicating that miRNA changes between brain and CSF/plasma samples do not necessarily run in parallel [22].

Among other targets, miR181c-5p and miR-210-3p have neuronal pentraxin 1 (NPTX1) and neuronal 
$\mathrm{MCl}$

A
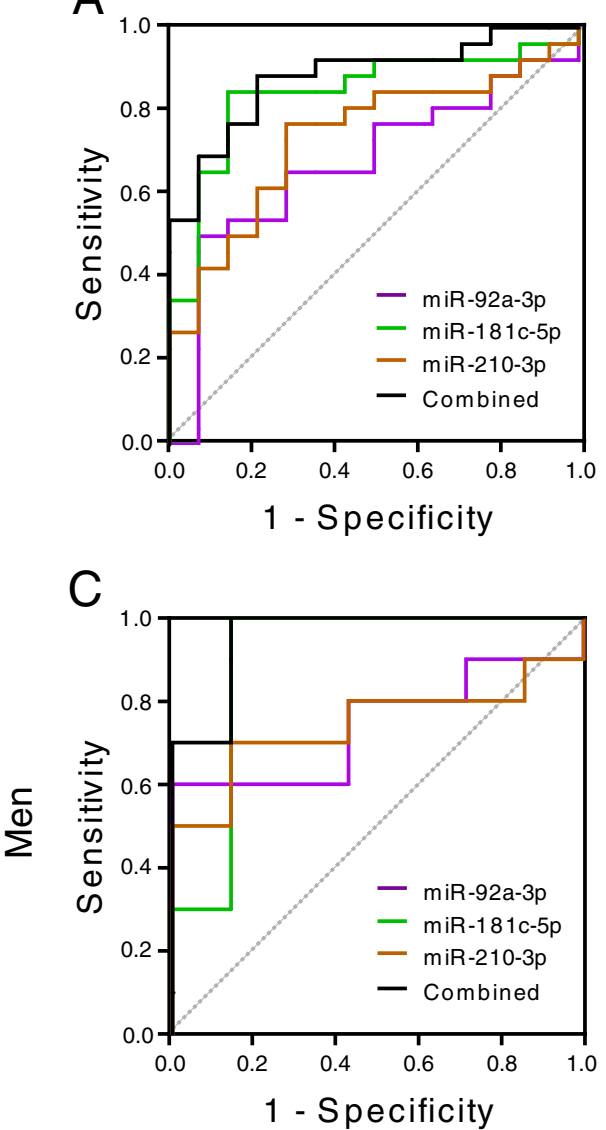

E

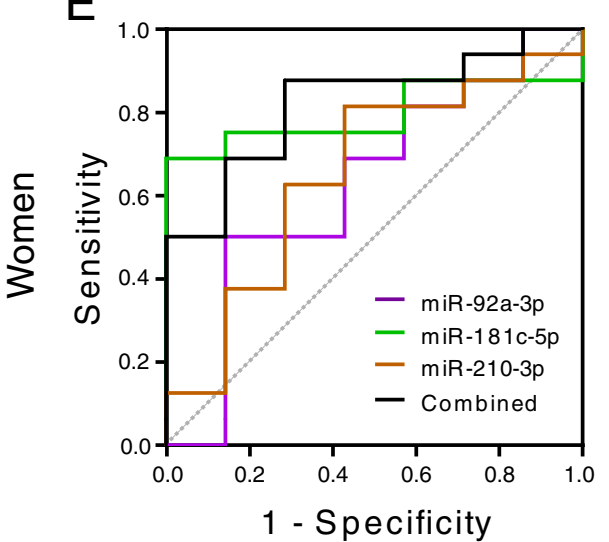

$A D$

B

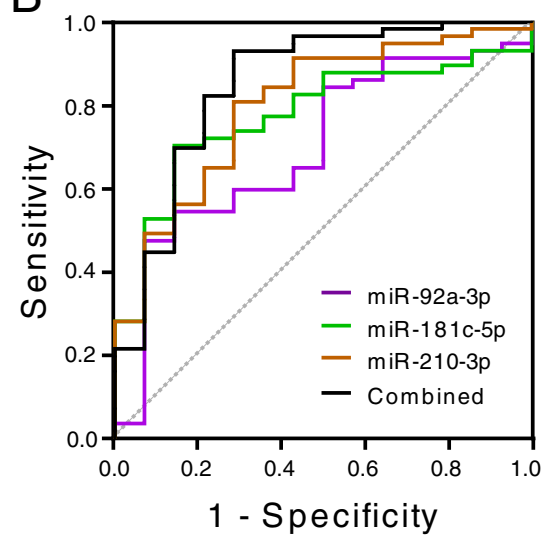

D

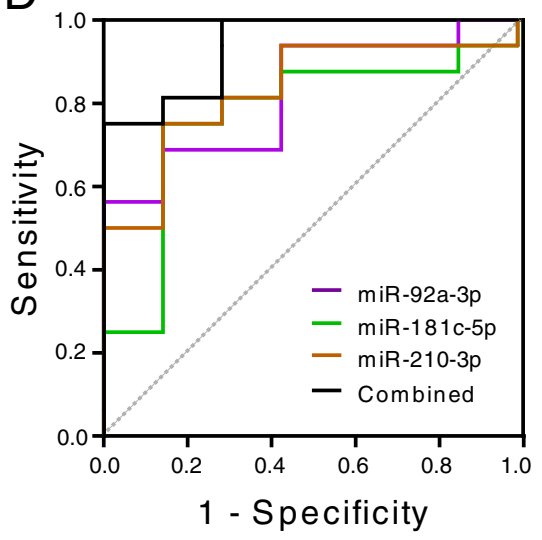

$\mathrm{F}$

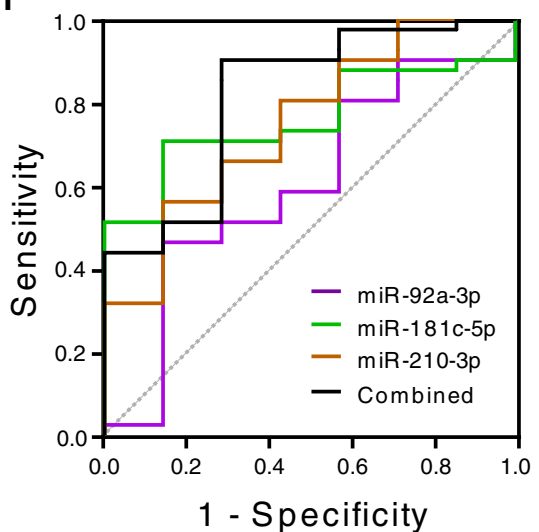

Fig. 2 miRNAs were combined to reach the best predictive value. ROC curve analysis for $\mathrm{MCl}(\mathbf{a})$ and $\mathrm{AD}$ (b) cases are shown. Values for men (c, d) and women $(\mathbf{e}, \mathbf{f})$ are represented separately

pentraxin receptor (NPTXR) as potential targets. Indeed, it has been described that miR-210-3p regulates the expression of NPTX1 and NPTXR [50]. These proteins are involved in the recruitment and clustering of AMPA receptors in postsynaptic terminals, organising and modelling the activity of glutamatergic synapses in the hippocampus affecting their synaptic plasticity [51, 52].
Thus, an increase of these miRNAs would reduce the levels of NPTX1 and NPTXR and could contribute to the glutamatergic synaptic dysfunction and early cognitive impairment present in $\mathrm{AD}$ prior to neurodegeneration. In fact, the increase in miR-181c-5p coincided with a decrease in NPTX1 and NPTXR protein in the entorhinal cortex. 


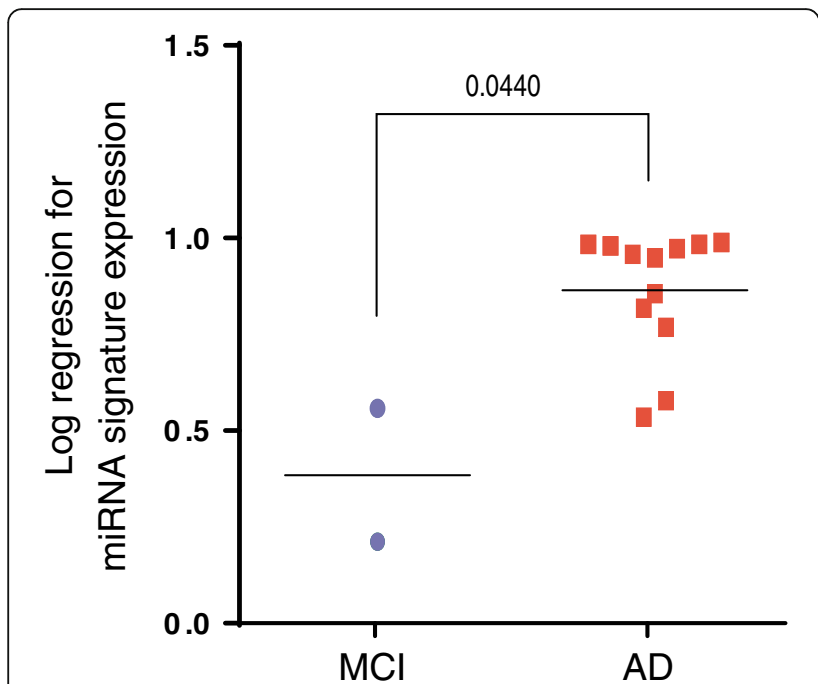

Fig. $3 \mathrm{MCl}$ patients were separated according to follow-up diagnosis. From 26 patients diagnosed as MCl in the first place, 2 remained stable and 12 have progressed to $A D$ (see Additional file 1: Table S3). miRNA expression levels were combined using log regression approach. Mean of miRNA signature values for each group is shown. Statistical significance was evaluated by the MannWhitney $U$ test. $P$ values $<0.05$ were considered statistically significant

Based on the observed increase in miR-92a-3p, miR-181c-5p and miR-210-3p, we propose that these miRNAs constitute a molecular signature that could be used as a biomarker for $\mathrm{MCI}$ and $\mathrm{AD}$. The AUC values of this molecular signature are similar (0.893 accuracy, $84.6 \%$ sensitivity and $85.71 \%$ sensitivity) to the ones obtained in other studies that have proposed either a 12-miRNA signature that distinguishes MCI with 0.842 accuracy, $81.1 \%$ specificity and $87.7 \%$ sensitivity in blood [29] or a 9-miRNAs serum signature for AD cases identification with an AUC value of $0.978,93.4 \%$ sensitivity and $98.8 \%$ of specificity [53]. Our results are the first that described an alteration of miR-92a-3p and miR-210-3p levels in biological fluids from $A D$ patients. Only a previous report showed that soluble $A \beta$ secreted from 7PA2 cells produced an increase in miR-210 levels in a mature primary culture of cortical neurons [54] and another study by Cogswell and co-workers reported that the levels of miR-92 were enhanced in AD hippocampus [47]. On the other hand, the observed increase in miR-181c-5p in plasma of MCI and AD subjects is challenged by previous reports reporting opposite observations. For instance, a decrease in miR-181c levels was reported in serum [20, 30, 53] and CSF [47] from AD patients. The causes of these discrepancies are currently unclear.

We were able to follow up for several years the evolution of the MCI patients included in our study, and we observed that the signature values in the plasma of the $\mathrm{MCI}$ patients that progressed to $\mathrm{AD}$ were significantly higher than the values found in the MCI patients that did not progress to dementia. Thus, plasma levels of miR-92a-3p, miR-181c-5p and miR-210-3p could not only be used as a biomarker signature for $\mathrm{MCI}$ and $\mathrm{AD}$ but might also indicate whether a patient with $\mathrm{MCI}$ will progress to AD. To our knowledge, this is the first study in which miRNAs levels in MCI patients are associated with the diagnosis of AD. However, we are aware that due to the low number of MCI patients that did not progress to $\mathrm{AD}$, further studies with more samples should be done before we can conclude that plasma levels of miR-92a-3p, miR-181c-5p and miR-210-3p are a good prognostic signature for the progression from MCI to AD.

Several studies have shown that AD-associated changes in miRNAs expression are also found in other types of dementia such as frontotemporal dementia or dementia with Lewy bodies [55]. Thus, those miRNAs could not be considered as eventual specific biomarkers
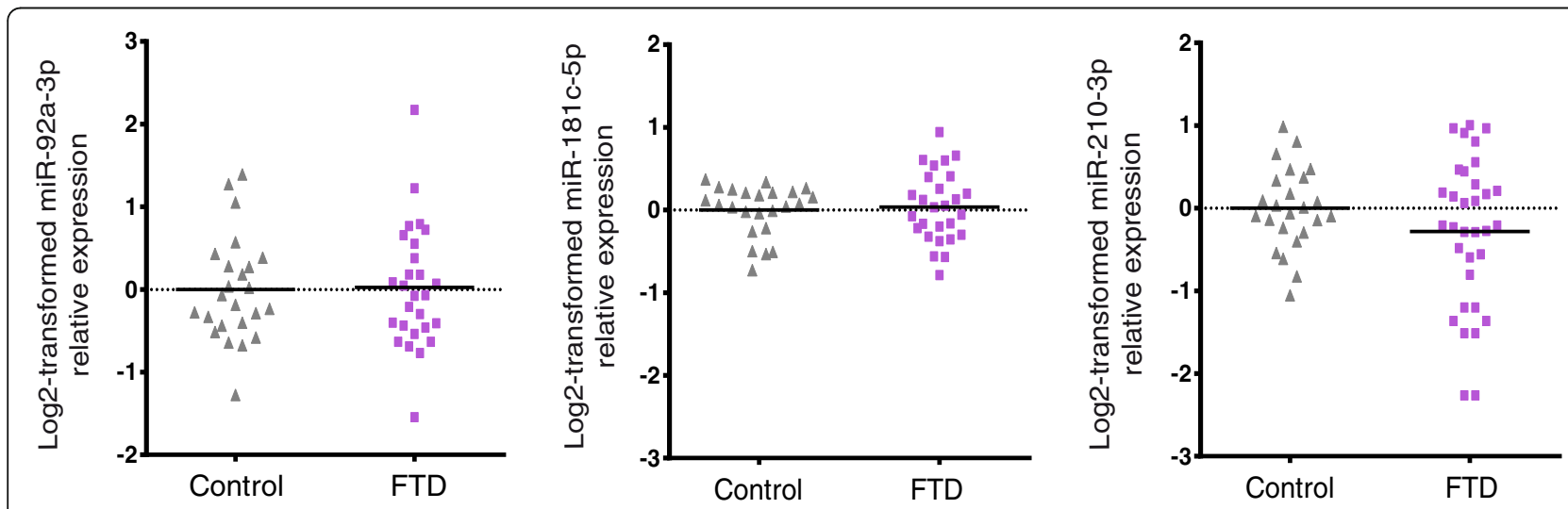

Fig. 4 Plasma miRNA levels in FTD compared to cognitively normal controls. Log2 transformed data were normalised versus the geometric mean of miR-191-5p and miR-484 levels. Statistical significance was evaluated by the Mann-Whitney $U$ test. $P$ values $<0.05$ were considered statistically significant 
for AD. For example, some reports have shown that miR-132 levels are downregulated in $\mathrm{AD}[46,49,56]$ and similar results were obtained in other neurodegenerative diseases $[19,55,57]$, suggesting that the alteration of this miRNA is linked to common pathological mechanisms in neurodegenerative diseases. In order to know whether this coincidence was also observed with miR-92a-3p, miR-181c-5p and miR-210-3p levels, we analysed their levels in plasma samples from a cohort of FTD patients. The results obtained indicate that no changes exist in our molecular miRNA signature between $\mathrm{HC}$ and FTD samples, suggesting that the increase in miR-92a-3p, miR-181c-5p and miR-210-3p levels could be specific for AD.

The existence of sex differences in $\mathrm{AD}$ is well established. Not only in the prevalence but also in the severity of the disease progression, being suggested that women could be more susceptible than men to the neuropathological cascade of $\mathrm{AD}$ [58]. Thus, it has become more necessary to introduce the effects of sex in the studies [59]. We have analysed whether differences exist between men and women and we did not find significant differences between sexes neither in the levels of the miRNAs nor in their value as a molecular signature. Only a slightly higher AUC was obtained in men to predict MCI (0.957 vs 0.821$)$ or $\mathrm{AD}(0.938$ vs 0.815$)$ compared to women. Thus, data from the studied cohort supports that our miRNA signature would be equally effective as a biomarker for $\mathrm{AD}$ in both men and women.

In summary, this study provides data supporting that a plasma miRNA signature composed of miR-92a-3p, miR-181c-5p and miR-210-3p could be useful as a non-invasive clinical biomarker for diagnosis of $\mathrm{AD}$ that could be used for improving current clinical trials and in future therapeutic strategies. Moreover, the levels of these miRNAs in MCI patients could predict the progression to $\mathrm{AD}$ dementia. Further studies are needed to assess whether changes in this miRNA signature correlates with known $A D$ biomarkers. Likewise, obtaining additional data from other $\mathrm{MCI}$ and $\mathrm{AD}$ cohorts would be necessary to fully validate the data presented in this study and to establish this miRNA signature as a reliable biomarker for early stages of $\mathrm{AD}$.

\section{Conclusions}

Deficits in synaptic function are likely to be involved in the development of $\mathrm{AD}$ in its preclinical stages. Here, we have analysed the levels of specific miRNAs related to synaptic proteins finding that the levels of miR-92a-3p, miR-181c-5p and miR-210-3p are deregulated in plasma from $\mathrm{MCI}$ and $\mathrm{AD}$ subjects while no changes are observed in plasma from FTD patients. Moreover, the analysis combining these three miRNAs yielded high diagnosis accuracy for distinguishing both MCI and AD subjects from healthy controls and could also predict $\mathrm{MCI}$ progress to $\mathrm{AD}$. Thus, we propose plasma levels of miR-92a-3p, miR-181c-5p and miR-210-3p as a molecular signature to be used as a novel biomarker for $\mathrm{AD}$.

\section{Additional file}

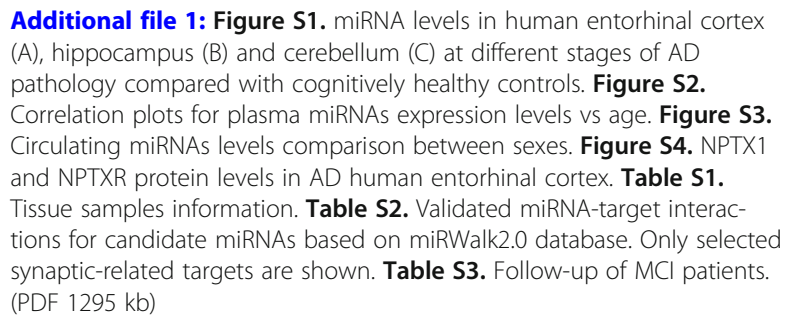

\section{Abbreviations}

AD: Alzheimer's disease; AUC: Area under the curve; CSF: Cerebrospinal fluid; FTD: Frontotemporal dementia; HC: Healthy controls; MCl: Mild cognitive impairment; miRNAs: MicroRNAs; NPTX1: Neuronal pentraxin 1;

NPTXR: Neuronal pentraxin receptor; ROC: Receiver operating characteristic; RT-qPCR: Quantitative real-time PCR

\section{Acknowledgements}

"Not applicable"

\section{Funding}

This work was supported by grants from Ministerio de Ciencia, Innovación y Universidades (SAF2014-59697-R; SAF2017-89271-R), Fundació La Marató TV3 (TV3-2014-3610), CIBERNED (CB06/05/0042 and PI2017/01) and Generalitat de Catalunya (SGR2014-0984 and SGR2017-749) to JRA and by the Department de Salut de la Generalitat de Catalunya, Pla Estratègic de Recerca I Innovació en Salut (SLT002/16/00408) to AL. DSW was supported by the Fundació La Marató TV3. JCS is a recipient of a Ph.D. Fellowship from the Ministerio de Ciencia, Innovación y Universidades. CF is a recipient of a Ph.D. Fellowship from the Department of Biochemistry and Molecular Biology of the Universitat Autònoma de Barcelona.

\section{Availability of data and materials}

The datasets used and/or analysed during the current study is available from the corresponding authors on reasonable request.

\section{Authors' contributions}

JRA and AJMM conceived and designed the study. DSW performed the experiments. $\mathrm{IH}, \mathrm{MB}$ and $\mathrm{AL}$ participated in the clinical diagnosis of subjects and the collection of serum. DSW, JCS and CF analysed the data. DSW, JRA and AJMM wrote the manuscript. CAS, JC and AL gave critical opinion and revised the written manuscript. All authors read and approved the final manuscript.

\section{Ethics approval and consent to participate}

All patients or their relatives gave written consent for plasma extraction and inclusion in the experimental cohorts. All clinical investigations were conducted according to the principles expressed in the Declaration of Helsinki, carried out according to the international Good Laboratory Practice (GLP) and Good Clinical Practice (GCP) standards and approved by the institution ethics committee.

Human brain tissue was provided by the Brain Bank of the Fundación CIEN, the BioBank of the Hospital Clinic-IDIBAPS and the Hospital Universitario Fundación Alcorcón following the guidelines of Spanish legislation.

\section{Consent for publication \\ "Not applicable"}

\section{Competing interests}

The authors declare that they have no potential competing interests.

\section{Publisher's Note}

Springer Nature remains neutral with regard to jurisdictional claims in published maps and institutional affiliations. 


\section{Author details}

${ }^{1}$ Institut de Neurociències and Dpt. Bioquímica i Biología Molecular, Universitat Autònoma de Barcelona, 08193 Cerdanyola del Vallès, Spain. ${ }^{2}$ Centro de Investigación Biomédica en Red sobre Enfermedades Neurodegenerativas (CIBERNED), Madrid, Spain. ${ }^{3}$ Research Center and Memory Clinic, Fundació ACE, Institut Català de Neurociències Aplicades, Universitat Internacional de Catalunya, Barcelona, Spain. ${ }^{4}$ Memory Unit, Department of Neurology, Institut d'Investigacions Biomèdiques Sant Pau Hospital de Sant Pau, Universitat Autònoma de Barcelona, Barcelona, Spain. ${ }^{5}$ Dominick P. Purpura Department of Neuroscience, Albert Einstein College of Medicine, New York, NY 10461, USA.

Received: 13 February 2019 Accepted: 28 April 2019 Published online: 15 May 2019

\section{References}

1. Polanco JC, Li C, Bodea LG, Martinez-Marmol R, Meunier FA, Götz J. Amyloid- $\beta$ and tau complexity-towards improved biomarkers and targeted therapies. Nat Rev Neurol. 2018;14:22-39.

2. Honig LS, Vellas B, Woodward M, Boada M, Bullock R, Borrie M, et al. Trial of solanezumab for mild dementia due to Alzheimer's disease. N Engl J Med. 2018;378:321-30.

3. Egan MF, Kost J, Tariot PN, Aisen PS, Cummings JL, Vellas B, et al. Randomized trial of Verubecestat for mild-to-moderate Alzheimer's disease. N Engl J Med. 2018;378:1691-703.

4. Lleó A, Cavedo E, Parnetti L, Vanderstichele H, Herukka SK, Andreasen N, et al. Cerebrospinal fluid biomarkers in trials for Alzheimer and Parkinson diseases. Nat Rev Neurol. 2015:11:41-55.

5. Blennow K, Mattsson N, Schöll M, Hansson O, Zetterberg H, et al. Amyloid biomarkers in Alzheimer's disease. Trends Pharmacol Sci. 2015;36:297-309.

6. Zetterberg H. Tau in bliofluids - relation to pathology, imaging and clinical features. Neuropathol Appl Neurobiol. 2015;43:194-9.

7. Jack CR Jr, Knopman DS, Jagust WJ, Petersen RC, Weiner MW, Aisen PS, et al. Tracking pathophysiological processes in Alzheimer's disease: an updated hypothetical model of dynamic biomarkers. Lancet Neurol. 2013;12:207-16.

8. Kadmiri NE, Said N, Slassi I, Moutawakil BE, Nadifi S, et al. Biomarkers for Alzheimer disease: classical and novel candidate's review. Neuroscience. 2018;370:181-90.

9. Frisoni GB, Boccardi M, Barkhof F, Blennow K, Cappa S, Chiotis K, et al. Strategic roadmap for an early diagnosis of Alzheimer's disease based on biomarkers. Lancet Neurol. 2017;16:661-76.

10. Forner S, Baglietto-Vargas D, Martini AC, Trujillo-Estrada L, LaFerla FM. Synaptic impairment in Alzheimer's disease: a dysregulated symphony. Trends Neurosci. 2017;40:347-57.

11. Miñano-Molina AJ, España J, Martin E, Barneda-Zahonero B, Fado R, Sole M, et al. Soluble oligomers of amyloid-beta peptide disrupt membrane trafficking of alpha-amino-3-hydroxy-5-methylisoxazole-4-propionic acid receptor contributing to early synapse dysfunction. J Biol Chem. 2011;286: 27311-21.

12. Roselli F, Hutzler $P$, Wegerich $Y$, Livrea $P$, Almeida OFX. Disassembly of shank and Homer synaptic clusters is driven by soluble $\beta$-amyloid ${ }_{1-40}$ through divergent NMDAR-dependent signalling pathways. PLoS One. 2009;4:e6011. https://doi.org/10.1371/journal.pone.0006011.

13. Reddy PH, Mani G, Park BS, Jacques J, Murdoch G, Whetsell W Jr, et al. Differential loss of synaptic proteins in Alzheimer's disease: implications for synaptic dysfunction. J Alzheimers Dis. 2005;7:103-17.

14. Casaletto KB, Elahi FM, Bettcher BM, Neuhaus J, Bendlin BB, Asthana S, et al. Neurogranin, a synaptic protein, is associated with memory independent of Alzheimer biomarkers. Neurology. 2017;89:1782-8.

15. Schratt G. microRNAs at the synapse. Nat Rev Neurosci. 2009;10:842-9.

16. Im HI, Kenny PJ. MicroRNAs in neuronal function and dysfunction. Trends Neurosci. 2012;35:325-34.

17. Lau P, Bossers K, Janky R, Salta E, Frigerio CS, Barbash S, et al. Alteration of the microRNA network during the progression of Alzheimer's disease. EMBO Mol Med. 2013;5:1613-34.

18. Hébert SS, Horré K, Nicolaï L, Bergmans B, Papadopoulou AS, Delacourte A, et al. MicroRNA regulation of Alzheimer's amyloid precursor protein expression. Neurobiol Dis. 2009;33:422-8.

19. Nelson PT, Wang WX. MiR-107 is reduced in Alzheimer's disease brain neocortex: validation study. J Alzheimers Dis. 2010;21:75-9.
20. Salta E, De Strooper B. microRNA-132: a key noncoding RNA operating in the cellular phase of Alzheimer's disease. FASEB J. 2017;31:424-33.

21. Geekiyanage $H$, Jicha GA, Nelson PT, Chan C. Blood serum miRNA: noninvasive biomarkers for Alzheimer disease. Exp Neurol. 2012;235:491-6.

22. Sala-Frigerio C, Pau P, Salta E, Tournoy J, Bossers K, Vandenberghe R, et al. Reduced expression of hsa-miR-27a-3p in CSF of patients with Alzheimer disease. Neurology. 2013;81:2103-6.

23. Müller M, Kuiperij HB, Claassen JA, Küsters B, Verbeek MM. MicroRNAs in Alzheimer's disease: differential expression in hippocampus and cell-free cerebrospinal fluid. Neurobiol Aging. 2014;35:152-8.

24. Denk J, Boelmans K, Siegismund C, Lassner D, Arlt S, Jahn H. MicroRNA profiling of CSF reveals potential biomarkers to detect Alzheimer's disease. PLoS One. 2015;10(5):e0126423.

25. Hara N, Kikuchi M, Miyashita A, Hatsuta H, Saito Y, Kasuga K, et al. Serum microRNA miR-501-3p as a potential biomarker related to the progression of Alzheimer's disease. Acta Neurophatol Commun. 2017;5:10. https://doi. org/10.1186/s40478-017-0414-z.

26. Kalani A, Tyagi A, Tyagi N. Exosomes: mediators of neurodegeneration, neuroprotection and therapeutics. Mol Neurobiol. 2014:49:590-600.

27. Riancho J, Vazquez-Higuera JL, Pozueta A, Lage C, Kazimierczak M, Bravo M, et al. MicroRNA profile in patients with Alzheimer's disease: analysis of miR9-5p and miR-598 in raw and exosome enriched cerebrospinal fluid samples. J Alzheimers Dis. 2017;57:483-91.

28. Zetterberg $\mathrm{H}$, Blennow K. From cerebrospinal fluid to blood: the third wave of fluid biomarkers for Alzheimer's disease. J Alzheimers Dis. 2018; 64:S271-9.

29. Sheinerman KS, Tsivinsky VG, Abdullah L, Crawford F, Umansky SR. Plasma microRNA biomarkers for detection of mild cognitive impairment: biomarker validation study. Aging. 2013;5:925-38.

30. Leidinger P, Backes C, Deutscher S, Schmitt K, Mueller SC, Frese KA. Blood based 12-miRNA signature of Alzheimer disease patients. Genome Biol. 2013;14:R78

31. Tan L, Yu JT, Tan MS, Liu QY, Wang HF, Zhang W. Genome-wide serum microRNA expression profiling identifies serum biomarkers for Alzheimer's disease. J Alzheimers Dis. 2014;40:1017-27.

32. Nagaraj S, Laskowska-Kaszub K, Debski KJ, Wojsiat J, Dabrowski M, Gabryelewicz T, et al. Profile of 6 microRNA in blood plasma distinguish early stage Alzheimer's disease patients from non-demented subjects. Oncotarget. 2017;8:16122-43.

33. Cosín-Tomás M, Antonell A, Lladó A, Alcolea D, Fortea J, Ezquerra M, Lleó A, et al. Plasma miR-34a-5p and miR-545-3p as early biomarkers of Alzheimer's disease: potential and limitations. Mol Neurobiol. 2017;54:5550-62.

34. IIlán-Gala I, Alcolea D, Montal V, Dols-Icardo O, Muñoz L, de Luna N, et al. CSF SAPP $\beta$, YKL-40, and NfL along the ALS-FTD spectrum. Neurology. 2018; 91:e1619-28.

35. Petersen RC, Smith GE, Waring SC, Ivnik RJ, Tangalos EG, Kokmen E. Mild cognitive impairment: clinical characterization and outcome. Arch Neurol. 1999;56:303-8.

36. Espinosa A, Alegret M, Valero S, Vinyes-Junque G, Hernandez I, Mauleon A et al. A longitudinal follow-up of 550 mild cognitive impairment patients: evidence for large conversion to dementia rates and detection of major risk factors involved. J Alzheimers Dis. 2013;34:769-80.

37. Rascovsky K, Hodges JR, Knopman D, Mendez MF, Kramer JH, Neuhaus J, et al. Sensitivity of revised diagnostic criteria for the behavioural variant of frontotemporal dementia. Brain. 2011;134:2456-77.

38. Gorno-Tempini ML, Hillis AE, Weintraub S, Kertesz A, Mendez M, Cappa SF, et al. Classification of primary progressive aphasia and its variants. Neurology. 2011;15:1006-14.

39. Brooks BR, Miller RG, Swash M, Munsat TL. El Escorial revisited: revised criteria for the diagnosis of amyotrophic lateral sclerosis. Amyotroph Lateral Scler Other Motor Neuron Disord. 2000;1(5):293-9.

40. Moldovan L, Batte KE, Trgovcich J, Wisler J, Marsh CB, Piper M. Methodological challenges in utilizing miRNAs as circulating biomarkers. J Cell Mol Med. 2014;18:371-90.

41. Fortunato $\mathrm{O}$, Boeri $\mathrm{M}$, Verri $\mathrm{C}$, Conte $\mathrm{D}$, Mensah $\mathrm{M}$, Suatoni $\mathrm{P}$, Pastorino $U$, et al. Assessment of circulating microRNAs in plasma of lung cancer patients. Molecules. 2014;19:3038-54.

42. Vennemann M, Koppelkamm A. Postmortem mRNA profiling II: practical considerations. Forensic Sci Int. 2010;203:76-82.

43. Pfaffl MW. A new mathematical model for relative quantification in real-time RT-PCR. Nucleic Acids Res. 2001;29:45e-45. 
44. Andersen $\mathrm{CL}$, Jensen JL, Ørntoft TF. Normalization of real-time quantitative reverse transcription-PCR data: a model-based variance estimation approach to identify genes suited for normalization, applied to bladder and color cancer data sets. Cancer Res. 2004;64:5245-50.

45. Vandesompele J, De Preter K, Pattyn F, Poppe B, Van Roy N, De Paepe A, et al. Accurate normalization of real-time quantitative RT-PCR data by geometric averaging of multiple internal control genes. Genome Biol. 2002; 3:34-1.

46. Cogswell JP, Ward J, Taylor IA, Waters M, She Y, Cannon B, et al. Identification of miRNA changes in Alzheimer's disease brain and CSF yields putative biomarkers and insights into disease pathways. J Alzheimers Dis. 2008;14:27-41.

47. Nunez-Iglesias J, Liu CC, Morgan TE, Finch CE, Zhou XJ. Joint genome-wide profiling of miRNA and mRNA expression in Alzheimer's disease cortex reveals altered miRNA regulation. PLoS One. 2010;5:e8898.

48. Wiedrick JT, Phillips JI, Lusardi TA, McFarland TJ, Lind B, Sandau US, et al. Validation of microRNA biomarkers dor Alzheimer disease in human cerebrospinal fluid. J Alzheimers Dis. 2019;67:875-91.

49. Pichler S, Gu W, Hartl D, Gasparoni G, Leidinger P, Keller A, et al. The miRNome of Alzheimer's disease: consistent down regulation of the miR132/212 cluster. Neurobiol Aging. 2016;167:e1-167 e10.

50. Pulkkinen K, Malm T, Turunen M, Koistinaho J, Ylä-Herttuala S. Hypoxia induces microRNA miR-210 in vitro and in vivo ephrin-A3 and neuronal pentraxin 1 are potentially regulated by miR-210. Have as a predicted target. FEBS Lett. 2008;582:2397-401.

51. Pelkey KA, Barksdale E, Craig MT, Yuan X, Sukumaran M, Vargish GA, et al. Pentraxins coordinante excitatory synapse maturation and circuit integration of parvalbumin interneurons. Neuron. 2015;85:1257-72.

52. Lee SJ, Wei M, Zhang C, Maxeiner S, Pak C, Calado Boteho S, et al. Presynaptic neuronal pentraxin receptor organizes excitatory and inhibitory synapses. J Neurosci. 2017;37:1062-80.

53. Guo R, Fan G, Zhang J, Wu C, Du Y, Ye H, et al. A 9-microRNA signature in serum serves as a noninvasive biomarker in early diagnosis of Alzheimer's disease. J Alzheimers Dis. 2017;60:1365-77.

54. Li JJ, Dolios G, Wang R, Liao FF. Soluble beta-amyloid peptides, but not insoluble fibrils, have specific effect on neuronal microRNA expression. PLoS One. 2014;9:e90770.

55. Hébert SS, Wang WX, Zhu Q, Nelson PT. A study of small RNAs from cerebral neocortex of pathology-verified Alzheimer's disease, dementia with Lewy bodies, hippocampal sclerosis, frontotemporal lobar dementia, and non-demented human controls. J Alzheimers Dis. 2013;35:335-48.

56. Zhu QB, Unmehopa U, Bossers K, Hu YT, Verwer R, Balesar R, et al. MicroRNA-132 and early growth response-1 in nucleus basalts of Meynert during the course of Alzheimer's disease. Brain. 2016;139:908-21.

57. Chen-Plotkin AS, Unger TL, Gallagher MD, Bill E, Kwong LK, Volpicelli-Daley $L$, et al. TMEM106B, the risk gene for frontotemporal dementia, is regulated by the microRNA-132/212 cluster and affects progranulin pathways. J Neurosci. 2012;32:11213-27.

58. Sinforiani E, Citterio A, Zucchella C, Bono G, Corbetta S, Merlo P, et al. Impact of gender differences on the outcome of Alzheimer's disease. Dementia. 2010;30:147-54.

59. Mazure CM, Swendsen J. Sex differences in Alzheimer's disease and other dementias. Lancet Neurol. 2016;15:451-2.

\section{Ready to submit your research? Choose BMC and benefit from:}

- fast, convenient online submission

- thorough peer review by experienced researchers in your field

- rapid publication on acceptance

- support for research data, including large and complex data types

- gold Open Access which fosters wider collaboration and increased citations

- maximum visibility for your research: over $100 \mathrm{M}$ website views per year

At BMC, research is always in progress.

Learn more biomedcentral.com/submissions 